\title{
Non-radial functions, nonlocal operators and Markov processes over $p$-adic numbers
}

\author{
Leonardo F. Chacón-Cortés ${ }^{1, *}$, Oscar F. Casas-Sánchez ${ }^{2}$
}

Edited by

Juan Carlos Salcedo-Reyes

(salcedo.juan@javeriana.edu.co)

1. Departamento de Matemáticas,

Pontificia Universidad Javeriana,

Cra. 7 N. 40-62, Bogotá, Colombia,

2. Escuela de Matemáticas y Estadística, Facultad de Ciencias, Universidad

Pedagógica y Tecnológica de Colombia,

Avenida Central del Norte 39-115,

Tunja, Boyacá, Colombia.

*leonardo.chacon@javeriana.edu.co

Received: 07-09-2018

Accepted: 10-05-2019

Published on line: 27-08-2019

Citation: Chacón-Cortés LF,

Casas-Sánchez OF. Non-radial functions,

nonlocal operators and Markov

processes over $p$-adic numbers,

Universitas Scientiarum, 24 (2): 381-406, 2019.

doi: 10.11144/Javeriana.SC24-2.nrfn

Funding:

N.A.

Electronic supplementary material: N.A.

OPEN ACCESS

\begin{abstract}
The main goal of this article is to study a new class of nonlocal operators and the Cauchy problem for certain parabolic-type pseudodifferential equations naturally associated with them. The fundamental solutions of these equations are transition functions of Markov processes on an $n$-dimensional vector space over the $p$-adic numbers. We also study some properties of these Markov processes, including the first passage time problem.
\end{abstract}

Keywords: Markov processes; non-Archimedean analysis; nonlocal operators; $p$-adic numbers.

\section{Introduction}

Markov processes on ultrametric spaces have been of strong interest in the last years due their connections with models of complex systems. These processes are very convenient to describe phenomena where the display of space states exhibits a hierarchical structure, see e.g., [1-16] and the references therein. Frauenfelder, Parisi, and Stain, among others [9, 15], proposed to describe the states of complex biological systems with a natural hierarchical organization using ultrametric spaces. Avetisov et al. have constructed models of ultrametric diffusion constrained by hierarchical energy landscapes, see [1-6]. From a mathematical point of view, the structure of the space of configuration states of a complex system is approximated by an ultrametric space $\mathbb{Q}_{p}$ (see Definition 2.1). On the other hand, the time-evolution of a complex system can be described by a parabolic-type pseudodifferential equation in $\mathbb{Q}_{p}$. This master equation controls the time-evolution of the transition function of a Markov process.

The first author et al. [17] introduced a class of nonlocal operators determined by radial functions and solved the associated Cauchy problem. Furthermore, they associated a Markov process to each operator and studied the related 
problem of the first return. This problem was studied first in dimension one by Avetisov, Bikulov and Zubarev [2]. Then the authors treated the same problem in dimensions 2, 3 and 4 for elliptic forms [18-21].

In this article, we introduce an operator class in arbitrary dimension, that unlike the one introduced in [17], is determined by non-radial functions and includes the elliptic operators studied in $[12,19,22]$ (see Definition 3.2). We also solve the Cauchy problem for the master equations attached to these operators (see Theorem 4.6). Moreover, we attach to each of these operators a Markov process (random walk) which is bounded and has no discontinuities other than jumps (see Theorem 5.8). Finally, we study the first passage time problem (see Theorem 6.9).

The article is organized as follows: In Section 2 we review the basic notions of $p$-adic analysis, and in Section 3 we introduce a new type of nonlocal operators. Given an operator, we study the Cauchy problem for its master equation in Section 4 and its Markov process in Section 5. Finally, Section 6 is devoted to the first passage time problem for the previous Markov process.

\section{Preliminaries}

In this section we collect the basic results on $p$-adic analysis which will be used in this work. For a detailed exposition on this theory the reader may consult $[11,23,24]$.

The field of $p$-adic numbers

Throughout this article $p$ will denote a prime number different from 2 . We consider the field of rational numbers $\mathbb{Q}$ endowed with the $p$-adic norm $|\cdot|_{p}$, which is defined as

$$
|x|_{p}= \begin{cases}0 & \text { if } x=0 \\ p^{-\gamma} & \text { if } x=p^{\gamma} \frac{a}{b},\end{cases}
$$

where $a$ and $b$ are integers coprime with $p$. The integer $\gamma:=\operatorname{ord}(x)$, with $\operatorname{ord}(0):=+\infty$, is called the $p$-adic order of $x$.

Definition 2.1. The field of $p$-adic numbers $\mathbb{Q}_{p}$ is defined as the completion of $\mathbb{Q}$ with respect to $|\cdot|_{p}$.

With an abuse of notation, we keep denoting by $|\cdot|_{p}$ and ord the natural extensions of the previous norm and order to $\mathbb{Q}_{p}$. In particular, the relation $|x|_{p}=p^{-\operatorname{ord}(x)}$ holds for any $x \in \mathbb{Q}_{p} \backslash\{0\}$. In addition, we extend the order 
and norm to $\mathbb{Q}_{p}^{n}$ by taking ord $(x):=\min _{1 \leqslant i \leqslant n}\left\{\operatorname{ord}\left(x_{i}\right)\right\}$ and

$$
\|x\|_{p}:=p^{-\operatorname{ord}(x)}=\max _{1 \leqslant i \leqslant n}\left|x_{i}\right|_{p}, \quad \text { for } x=\left(x_{1}, \ldots, x_{n}\right) \in \mathbb{Q}_{p}^{n} .
$$

A non-zero $p$-adic number $x$ has a unique representation as a Laurent series in $p$ given by

$$
x=p^{\operatorname{ord}(x)} \sum_{j=0}^{\infty} x_{j} p^{j}, \quad \text { where } x_{j} \in\{0,1,2, \ldots, p-1\}, x_{0} \neq 0 .
$$

Using this expansion, we define the fractional part $\{x\}_{p}$ of $x \in \mathbb{Q}_{p}$, as the rational number

$$
\{x\}_{p}= \begin{cases}0 & \text { if } x=0, \text { or } \operatorname{ord}(x) \geqslant 0 ; \\ p^{\operatorname{ord}(x)} \sum_{j=0}^{-\operatorname{ord}(x)-1} x_{j} p^{j} & \text { if } \operatorname{ord}(x)<0 .\end{cases}
$$

For $\gamma \in \mathbb{Z}$, we write $B_{\gamma}^{n}(a)=\left\{x \in \mathbb{Q}_{p}^{n}:\|x-a\|_{p} \leqslant p^{\gamma}\right\}=B_{\gamma}^{1}\left(a_{1}\right) \times \cdots \times B_{\gamma}^{1}\left(a_{n}\right)$ for the ball of radius $p^{\gamma}$ with center at $a=\left(a_{1}, \ldots, a_{n}\right) \in \mathbb{Q}_{p}^{n}$. We also write $B_{\gamma}^{n}(0):=B_{\gamma}^{n}$. Note that $B_{0}^{n}(0)=\mathbb{Z}_{p}^{n}$, is the product of $n$ copies of the ring of p-adic integers $\mathbb{Z}_{n}$. If $A \subseteq \mathbb{Q}^{n}$ we denote by $1_{A}(x)$ the characteristic function of $A$. If $A=B_{0}^{n}$ we write $\Omega\left(\|x\|_{p}\right)=1_{B_{0}^{n}}(x)$ instead.

\section{The Bruhat-Schwartz space}

A complex-valued function $\varphi$ defined on $\mathbb{Q}_{p}^{n}$ is called locally constant if for any $x \in \mathbb{Q}_{p}^{n}$ there exist an integer $l(x) \in \mathbb{Z}$ such that

$$
\varphi\left(x+x^{\prime}\right)=\varphi(x) \text { for } x^{\prime} \in B_{l(x)}^{n} .
$$

A function $\varphi: \mathbb{Q}_{p}^{n} \rightarrow \mathbb{C}$ is called a Brubat-Schwartz function (or a test function) if it is locally constant with compact support. The $\mathbb{C}$-vector space of Bruhat-Schwartz functions is denoted by $S\left(\mathbb{Q}_{p}^{n}\right):=S$. For $\varphi \in S\left(\mathbb{Q}_{p}^{n}\right)$, the largest of such numbers $l=l(\varphi)$ satisfying (1) is called the exponent of local constancy of $\varphi$. The set of all distributions (linear functionals) on $S\left(\mathbb{Q}_{p}^{n}\right)$ is denoted by $S^{\prime}\left(\mathbb{Q}_{p}^{n}\right):=S^{\prime}$.

Set $\chi_{p}(y)=\exp \left(2 \pi i\{y\}_{p}\right)$ for $y \in \mathbb{Q}_{p}$. The map $\chi_{p}(\cdot)$ is an additive character on $\mathbb{Q}_{p}$, i.e. a continuous map from $\mathbb{Q}_{p}$ into the unit circle satisfying that $\chi_{p}\left(y_{0}+y_{1}\right)=\chi_{p}\left(y_{0}\right) \chi_{p}\left(y_{1}\right)$, for $y_{0}, y_{1} \in \mathbb{Q}_{p}$.

Given $\xi=\left(\xi_{1}, \ldots, \xi_{n}\right)$ and $x=\left(x_{1}, \ldots, x_{n}\right) \in \mathbb{Q}_{p}^{n}$, the Fourier transform of 
$\varphi \in S\left(\mathbb{Q}_{p}^{n}\right)$ is defined as

$$
\left(\mathscr{F}_{x \rightarrow \xi} \varphi\right)=\widehat{\varphi}(\xi)=(\mathscr{F} \varphi)(\xi)=\int_{\mathbb{Q}_{p}^{n}} \chi_{p}(\xi \cdot x) \varphi(\xi) \mathrm{d}^{n} x, \quad \text { for } \xi \in \mathbb{Q}_{p}^{n},
$$

where $\xi \cdot x:=\sum_{j=1}^{n} \xi_{j} x_{j}$, and $\mathrm{d}^{n} x$ is the Haar measure on $\mathbb{Q}_{p}^{n}$ normalized by the condition $\operatorname{Vol}\left(B_{0}^{n}\right)=1$. The Fourier transform is a linear isomorphism from $S\left(\mathbb{Q}_{p}^{n}\right)$ onto itself satisfying $(\mathscr{F}(\mathscr{F} \varphi))(\xi)=\varphi(-\xi)$.

The Fourier transform $\mathscr{F}[f]$ of a distribution $f \in S^{\prime}\left(\mathbb{Q}_{p}^{n}\right)$ is defined by

$$
(\mathscr{F}[f], \varphi)=(f, \mathscr{F}(\varphi)) \text {, for all } \varphi \in S\left(\mathbb{Q}_{p}^{n}\right) .
$$

The Fourier transform $f \mapsto \mathscr{F}[f]$ is a linear isomorphism from $S^{\prime}\left(\mathbb{Q}_{p}^{n}\right)$ onto $S^{\prime}\left(\mathbb{Q}_{p}^{n}\right)$. Moreover, $f=\mathscr{F}[\mathscr{F}[f](-\xi)]$.

\section{A New Class of Nonlocal operators over $\mathbb{Q}_{p}^{n}$}

Let $\widetilde{\mathscr{E}}$ be the $\mathbb{C}$-vector space of all functions $\varphi: \mathbb{Q}_{p}^{n} \rightarrow \mathbb{C}$, such that there exists a ball $B_{l}^{n}$, with $l$ depending only on $\varphi$, and $\varphi\left(x+x^{\prime}\right)=\varphi(x)$ for any $x^{\prime} \in B_{l}^{n}$. Notice that $S\left(\mathbb{Q}_{p}^{n}\right) \subset \tilde{\mathscr{E}}$. We identify any element of $\tilde{\mathscr{E}}$ with a distribution on $\mathbb{Q}_{p}^{n}$. We now recall the following fact: $T \in S^{\prime}$ with $\operatorname{supp}(T) \subset B_{N}^{n}$ if and only if $\widehat{T} \in \widetilde{\mathscr{E}}$ and its parameter of local constancy is greater than or equal to $-N$, cf. [11, p. 109] or [24, Proposition 3.17].

Definition 3.1. Let $g(\xi) \in \mathbb{Q}_{p}^{n}\left[\xi_{1}, \ldots, \xi_{n}\right]$ be a non-constant polynomial. We say that $g(\xi)$ is an elliptic polynomial of degree $d$, if it satisfies the following two conditions: (i) the polynomial $g(\xi)$ is homogeneous of degree $d$, and (ii) $g(\xi)=0$ if and only if $\xi=0$.

We note that if $g(\xi)$ is elliptic, then $c g(\xi)$ is also elliptic for any $c \in \mathbb{Q}_{p}^{*}$. Furthermore, there are infinitely many elliptic polynomials cf. [25, Lemma 24].

Definition 3.2. Let $\mathfrak{A}\left(\mathbb{Q}_{p}^{n}\right)$ denote the subset of all continuous functions $f: \mathbb{Q}_{p}^{n} \rightarrow \mathbb{Q}_{p}$, that satisfy the following conditions: (i) $f$ is a homogeneous function of degree $d \geqslant 1, d \in \mathbb{N}$; (ii) $f(\xi)=0$ if and only if $\xi=0$.

Note that if $g(\xi)$ is an elliptic polynomial of degree $d$, then $g(\xi) \in \mathfrak{A}\left(\mathbb{Q}_{p}^{n}\right)$.

Example 3.3. Let $\lambda, \mu \in \mathbb{Q}_{p}^{*}$ be different and not squares. Then $f_{\beta}: \mathbb{Q}_{p}^{2} \rightarrow \mathbb{Q}_{p}$ given by 


$$
f_{\beta}(x, y)= \begin{cases}\frac{\left(x^{2}-\lambda y^{2}\right)^{\beta}}{x^{2}-\mu y^{2}} & \text { if }(x, y) \neq(0,0) \\ 0 & \text { if }(x, y)=(0,0)\end{cases}
$$

belongs to $\mathfrak{A}\left(\mathbb{Q}_{p}^{2}\right)$ for any $\beta>1, \beta \in \mathbb{N}$. In general if $f_{1}(x), f_{2}(x) \in \mathfrak{A}\left(\mathbb{Q}_{p}^{n}\right)$ with degrees of homogeneity $d_{1}$ and $d_{2}$, respectively, and $d_{1}>d_{2}$, then the function $g_{\beta}: \mathbb{Q}_{p}^{n} \rightarrow \mathbb{Q}_{p}$ given by

$$
g_{\beta}(x)= \begin{cases}\left(\frac{f_{1}(x)}{f_{2}(x)}\right)^{\beta} & \text { if } x \neq 0, \\ 0 & \text { if } x=0\end{cases}
$$

belongs to $\mathfrak{A}\left(\mathbb{Q}_{p}^{n}\right)$ for each $\beta>1, \beta \in \mathbb{N}$. It is also easy to see that if $f_{1}(x)$, $f_{2}(x) \in \mathfrak{A}\left(\mathbb{Q}_{p}^{n}\right)$ and $\lambda \in \mathbb{Z}$ is a quadratic non-residue module $p$, then the functions $f_{1}^{2 \beta d_{2}}(x)-\lambda f_{2}^{2 \beta d_{1}}(x), f_{1}(x) f_{2}(x) \in \mathfrak{A}\left(\mathbb{Q}_{p}^{n}\right)$ for any integer $\beta \geqslant 1$.

Lemma 3.4. If $f \in \mathfrak{A}\left(\mathbb{Q}_{p}^{n}\right)$, there exist positive constants $A_{1}, A_{2}$, such that

$$
A_{1}\|\xi\|_{p}^{d} \leqslant|f(\xi)|_{p} \leqslant A_{2}\|\xi\|_{p}^{d}, \quad \text { for any } \xi \in \mathbb{Q}_{p}^{n} .
$$

Proof. The result is clear for $\xi=0$. Let $\xi \neq 0$, we write $\xi=p^{\gamma} \xi_{0}$, with $\left\|\xi_{0}\right\|_{p}=1$ and $\gamma=\operatorname{ord}(\xi)$. Since $f$ is homogeneous of degree $d$, then

$$
|f(\xi)|_{p}=\left|p^{d \gamma} f\left(\xi_{0}\right)\right|_{p}=\|\xi\|_{p}^{d}\left|f\left(\xi_{0}\right)\right|_{p} .
$$

Since $|f|_{p}$ is continuous and $U:=\left\{x \in \mathbb{Q}_{p}^{n} \mid\|x\|_{p}=1\right\}$ is a compact subset, we have that $A_{1}:=\inf _{\xi_{0} \in U}\left|f\left(\xi_{0}\right)\right|_{p}, A_{2}:=\sup _{\xi_{0} \in U}\left|f\left(\xi_{0}\right)\right|_{p}$, and this values are attained on $U$, for this reason,

$$
A_{1}\|\xi\|_{p}^{d} \leqslant|f(\xi)|_{p}=\|\xi\|_{p}^{d}\left|f\left(\xi_{0}\right)\right|_{p} \leqslant A_{2}\|\xi\|_{p}^{d} .
$$

Given $\varphi \in S$, positive constants $k, \alpha$ and a function $f \in \mathfrak{A}\left(\mathbb{Q}_{p}^{n}\right)$, we define

$$
\left(\mathbf{W}_{f} \varphi\right)(x)=-k \int_{\mathbb{Q}_{p}^{n}} \frac{\varphi(x-y)-\varphi(x)}{|f(y)|_{p}^{\alpha+\frac{n}{d}}} \mathrm{~d}^{n} y .
$$

When $f$ is an elliptic quadratic form and $n=2,3$ and 4 , the operator $\mathbf{W}_{f}$ was studied in [19] and [22]. These operators are well-defined because the 
inequalities in (2) and the continuity of $|f|_{p}$ imply that

$$
\int_{\|y\|_{p} \geqslant p^{l}} \frac{\mathrm{d}^{n} y}{|f(y)|_{p}^{\alpha+\frac{n}{d}}}<\infty
$$

for any $l \in \mathbb{Z}$ and any $\alpha>0$.

Lemma 3.5. The linear operator $\mathbf{W}_{f}: \widetilde{\mathscr{E}} \rightarrow \widetilde{\mathscr{E}}$ is well-defined. Moreover, from $\mathbf{W}_{f}$ arises a pseudodifferential operator, namely

$$
\mathscr{F}\left(\mathbf{W}_{f} \varphi\right)(\xi)=k\left(\int_{\mathbb{Q}_{p}^{n}} \frac{1-\chi_{p}(y \cdot \xi)}{|f(y)|_{p}^{\alpha+\frac{n}{d}}} \mathrm{~d}^{n} y\right)(\mathscr{F} \varphi)(\xi) .
$$

Proof. Let $\varphi \in \widetilde{\mathscr{E}}$ and $l$ its parameter of locally constancy. Note that

$$
\left(\mathbf{W}_{f} \varphi\right)(x)=k \frac{1_{\mathbb{Q}_{p}^{n} \backslash B_{l}^{n}}(x)}{|f(x)|_{p}^{\alpha+\frac{n}{d}}} * \varphi(x)+k \varphi(x) \int_{\|y\|_{p} \geqslant p^{l}} \frac{\mathrm{d}^{n} y}{|f(y)|_{p}^{\alpha+\frac{n}{d}}} .
$$

Thus, by using taking Fourier transform (in $S^{\prime}$ ) we obtain

$$
\mathscr{F}\left(\mathbf{W}_{f} \varphi\right)(\xi)=k\left(\int_{\mathbb{Q}_{p}^{n}} 1_{\mathbb{Q}_{p}^{n} \backslash B_{l}^{n}}(x) \frac{\left(1-\chi_{p}(x \cdot \xi)\right)}{|f(x)|_{p}^{\alpha+\frac{n}{d}}} \mathrm{~d}^{n} x\right)(\mathscr{F} \varphi)(\xi) .
$$

Since $\operatorname{supp}(\mathscr{F} \varphi) \subset B_{-l}^{n}$ and $\mathscr{F} \varphi \in S^{\prime}$, it follows that

$$
\mathscr{F}\left(\mathbf{W}_{f} \varphi\right)(\xi)=k\left(\int_{\mathbb{Q}_{p}^{n}} \frac{\left(1-\chi_{p}(x \cdot \xi)\right)}{|f(x)|_{p}^{\alpha+\frac{n}{d}}} \mathrm{~d}^{n} x\right) \mathscr{F} \varphi(\xi) .
$$

Remark 1. Let $\varphi \in S\left(\mathbb{Q}_{p}^{n}\right) \subset L^{\rho}\left(\mathbb{Q}_{p}^{n}\right)$, with $1 \leqslant \rho \leqslant \infty$. By Young's inequality the first term on the right-hand side of (5) belongs to $L^{\rho}\left(\mathbb{Q}_{p}^{n}\right)$, and by (2) the second term in (5) also belongs to $L^{p}\left(\mathbb{Q}_{p}^{n}\right)$. Thus $\mathbf{W}_{f}: S\left(\mathbb{Q}_{p}^{n}\right) \rightarrow L^{p}\left(\mathbb{Q}_{p}^{n}\right)$ is a well-defined linear operator and gives rise to a pseudodifferential operator,

$$
\left(\mathbf{W}_{f} \varphi\right)(x)=k \mathscr{F}_{\xi \rightarrow x}^{-1}\left(A_{f}(\xi) \mathscr{F}_{x \rightarrow \xi} \varphi\right)
$$

where

$$
A_{f}(\xi):=\int_{\mathbb{Q}_{p}^{n}} \frac{1-\chi_{p}(y \cdot \xi)}{|f(y)|_{p}^{\alpha+\frac{n}{d}}} \mathrm{~d}^{n} y
$$


Corollary 3.6. For $\alpha>0, A_{f}(\xi)$ is a negative function with associated Levy measure $d \mathscr{L}^{\alpha}(y)=|f(y)|_{p}^{-\alpha-\frac{n}{d}} \mathrm{~d}^{n}(y)$.

Proof. See [26, Lemma 18.18].

A similar representation for $A_{f}(x)=\|x\|^{\alpha}, \alpha>0$, was found by Kochubei, see [16, Proposition 2.3].

Some additional results

Lemma 3.7. There exist positive constants $C_{1}, C_{2}$, such that

$$
C_{1}\|\xi\|_{p}^{\alpha d} \leqslant A_{f}(\xi) \leqslant C_{2}\|\xi\|_{p}^{\alpha d}, \quad \text { for any } \xi \in \mathbb{Q}_{p}^{n} .
$$

Proof. We write $\xi=p^{\gamma} \xi_{0}$, with $\gamma=\operatorname{ord}(\xi)$ and $\left\|\xi_{0}\right\|_{p}=1$. Then

$$
A_{f}(\xi)=\int_{\mathbb{Q}_{p}^{n}} \frac{1-\chi_{p}\left(p^{\gamma} y \cdot \xi_{0}\right)}{|f(y)|_{p}^{\alpha+\frac{n}{d}}} \mathrm{~d}^{n} y .
$$

By making the following change of variables

$$
\begin{cases}z & =p^{\gamma} y \\ \mathrm{~d}^{n} z & =p^{-n \gamma} \mathrm{d}^{n} y\end{cases}
$$

and using the homogeneity of $f$, one gets

$$
A_{f}(\xi)=\|\xi\|_{p}^{\alpha d} \int_{\mathbb{Q}_{p}^{n}} \frac{1-\chi_{p}\left(z \cdot \xi_{0}\right)}{|f(z)|_{p}^{\alpha+\frac{n}{d}}} \mathrm{~d}^{n} z .
$$

Thus, we have that

$$
C_{1}\|\xi\|_{p}^{\alpha d} \leqslant A_{f}(\xi) \leqslant C_{2}\|\xi\|_{p}^{\alpha d},
$$

where

$$
C_{1}=\inf _{\xi_{0} \in \mathbb{Z}_{p}^{n}}\left(\int_{\mathbb{Q}_{p}^{n}} \frac{1-\chi_{p}\left(z \cdot \xi_{0}\right)}{|f(z)|_{p}^{\alpha+\frac{n}{d}}} \mathrm{~d}^{n} z\right),
$$




$$
C_{2}=\sup _{\xi_{0} \in \mathbb{Z}_{p}^{n}}\left(\int_{\mathbb{Q}_{p}^{n}} \frac{1-\chi_{p}\left(z \cdot \xi_{0}\right)}{|f(z)|_{p}^{\alpha+\frac{n}{d}}} \mathrm{~d}^{n} z\right)
$$

Remark 2. The function $A_{f}$ satisfies $A_{f}(t \xi)=|t|_{p}^{\alpha d} A_{f}(\xi)$ for all $t \in \mathbb{Q}_{p}^{*}$. Furthermore $A_{f}(\xi)=0$ if and only if $\xi=0$.

The following corollary will be very useful when we use Lebesgue's Dominated Convergence Theorem.

Corollary 3.8. If $\beta \geqslant 0$, then

$$
\int_{\mathbb{Q}_{p}^{n}}\|\xi\|_{p}^{\beta \rho} e^{-t \rho k A_{f}(\xi)} \mathrm{d}^{n} \xi \leqslant C t^{\frac{-(\beta \rho+n)}{\alpha d}}, \text { for } 1 \leqslant \rho<\infty \text { and } t>0 .
$$

Proof. By Lemma 3.7

$$
I:=\int_{\mathbb{Q}_{p}^{n}}\|\xi\|_{p}^{\beta \rho} e^{-\rho k t A_{f}(\xi)} \mathrm{d}^{n} \xi \leqslant \int_{\mathbb{Q}_{p}^{n}}\|\xi\|_{p}^{\beta \rho} e^{-C_{1} \rho k t\|\xi\|_{p}^{\alpha d}} \mathrm{~d}^{n} \xi .
$$

Let $m$ be an integer such that $p^{m-1} \leqslant t^{\frac{1}{\alpha d}} \leqslant p^{m}$, then

$$
I \leqslant \int_{\mathbb{Q}_{p}^{n}}\|\xi\|_{p}^{\beta \rho} e^{-C_{1} \rho k\left\|p^{-(m-1)} \xi\right\|_{p}^{\alpha d}} \mathrm{~d}^{n} \xi
$$

now by changing variables using $z=p^{-(m-1)} \xi$, we have

$$
I \leqslant C(k, \rho, \beta) t^{\frac{-(\beta \rho+n)}{\alpha d}}, \quad \text { for } t>0 .
$$

\section{Heat Kernels}

In this section we study the asymptotic properties for the heat kernels.

For $t>0$ and $x \in \mathbb{Q}_{p}^{n}$ we define a heat kernel as the function

$$
Z(x, t ; f, k):=Z(x, t)=\int_{\mathbb{Q}_{p}^{n}} \chi_{p}(-x \cdot \xi) e^{-k t A_{f}(\xi)} \mathrm{d}^{n} \xi .
$$

By Corollary 3.8, $Z(x, t)=\mathscr{F}_{\xi \rightarrow x}^{-1}\left[e^{-k t A_{f}(\xi)}\right] \in L^{1}\left(\mathbb{Q}_{p}^{n}\right) \cap L^{2}\left(\mathbb{Q}_{p}^{n}\right)$ for $t>0$. When considering $Z(x, t)$ as a function of $x$ for $t$ fixed we will write $Z_{t}(x)$. 
Theorem 3.9. $Z(x, t)$ satisfies the following properties:

(i) $Z(x, t) \leqslant C(n, p) t^{-\frac{n}{\alpha d}}$, for any $t>0$;

(ii) $\int_{\mathbb{Q}_{p}^{n}} Z(x, t) \mathrm{d}^{n} x=1$, for any $t>0$;

(iii) $Z_{t}(x) \in C\left(\mathbb{Q}_{p}^{n}, \mathbb{R}\right) \cap L^{1}\left(\mathbb{Q}_{p}^{n}\right) \cap L^{2}\left(\mathbb{Q}_{p}^{n}\right)$, for any $t>0$;

(iv) $Z_{t}(x) * Z_{t^{\prime}}(x)=Z_{t+t^{\prime}}(x)$, for any $t^{\prime}, t>0$;

(v) $\lim _{t \rightarrow 0^{+}} Z(x, t)=\delta(x)$ in $S^{\prime}\left(\mathbb{Q}_{p}^{n}\right)$.

Proof. (i) It follows from Lemma 3.7 and Lemma 3.8. For the proof of other properties see [18, Theorem 4.3].

\section{The Cauchy Problem}

In this section we use the theory of semi-groups on Hilbert spaces to solve the inhomogeneous and homogeneous Cauchy problems associated with the operator $\mathbf{W}_{f}$.

Proposition 4.1. The operator $\mathbf{W}_{f}$ satisfies the properties

(i) $\left(\mathbb{W}_{f} \varphi\right)(x)=k \mathscr{F}_{\xi \rightarrow x}^{-1}\left(A_{f}(\xi) \mathscr{F}_{x \rightarrow \xi} \varphi\right)$ for $\varphi \in S\left(\mathbb{Q}_{p}^{n}\right)$, and the image $\mathbf{W}_{f} \varphi \in C\left(\mathbb{Q}_{p}^{n}\right) \cap L^{p}\left(\mathbb{Q}_{p}^{n}\right)$ for $1 \leqslant \rho \leqslant \infty$. The operator $\mathbf{W}_{f}$ extends to an unbounded and densely defined operator in $L^{2}\left(\mathbb{Q}_{p}^{n}\right)$ with domain

$$
\operatorname{Dom}\left(\mathbf{W}_{f}\right)=\left\{\varphi \in L^{2}: A_{f}(\xi) \mathscr{F} \varphi \in L^{2}\right\}
$$

(ii) $\left(-\mathbf{W}_{f}, \operatorname{Dom}\left(\mathbf{W}_{f}\right)\right)$ is positive and self-adjoint operator.

(iii) $\mathbf{W}_{f}$ is the infinitesimal generator of a contraction $C_{0}$ bounded semigroup $(\mathscr{T}(t))_{t \geqslant 0}$.

Proof. (i) It follows from Lemma 3.5, Remark 1 and the fact that $A_{f}(\xi)$ is continuous, c.f. Lemma 3.7. Property (ii) follows from the fact that the Fourier transform preserves the inner product of $L^{2}$ and that the $\mathbf{W}_{f}$ is a pseudodifferential operator. (iii) See e.g., [27, Chap. 2, Sect. 3] or [28]. 
Consider the following Cauchy problem:

$$
\begin{cases}\frac{\partial u}{\partial t}(x, t)+\left(\mathbf{W}_{f} u\right)(x, t)=0, & x \in \mathbb{Q}_{p}^{n}, t \in[0, \infty), \\ u(x, 0)=u_{0}(x), & u_{0}(x) \in \operatorname{Dom}\left(\mathbf{W}_{f}\right),\end{cases}
$$

where $\left(\mathbf{W}_{f} \phi\right)(x)=k \mathscr{F}_{\xi \rightarrow x}^{-1}\left(A_{f}(\xi) \mathscr{F}_{x \rightarrow \xi} \phi\right)$ for $\phi \in \operatorname{Dom}\left(\mathbf{W}_{f}\right)$, see (6), and $u: \mathbb{Q}_{p}^{n} \times[0, \infty) \rightarrow \mathbb{C}$ is an unknown function. We say that a function $u(x, t)$ is a solution of $(7)$, if $u(x, t) \in C^{1}\left([0, \infty), L^{2}\left(\mathbb{Q}_{p}^{n}\right)\right) \cap C\left([0, \infty), \operatorname{Dom}\left(\mathbb{W}_{f}\right)\right)$ and $u$ satisfies (7) for all $t \geqslant 0$.

In this section, we understand the notions of continuity in $t$, differentiability in $t$, and equalities in the $L^{2}\left(\mathbb{Q}_{p}^{n}\right)$ sense, as it is customary in the semigroup theory.

From Proposition 4.1 we have that the operator $\mathbf{W}_{f}$ generates a $C_{0}$ semigroup $(\mathscr{T}(t))_{t \geqslant 0}$, so the Cauchy problem (7) is uniquely solvable with the solution continuously dependent on the initial data, and its solution is given by $u(x, t)=\mathscr{T}(t) u_{0}(x)$, for $t \geqslant 0$, see e.g., [28, Theorem 3.1.1]. However the general theory does not give an explicit formula for the semigroup $(\mathscr{T}(t))_{t \geqslant 0}$. In order to prove that for $t>0$ the operator $\mathscr{T}(t)$ coincides with the operator of convolution with the heat kernel $Z_{t} * \cdot$, we first construct, without using the semigroup theory, a solution of Cauchy problem (7) with the initial value $u_{0}(x) \in S\left(\mathbb{Q}_{p}^{n}\right)$. Then we extend the result to all initial values from $\operatorname{Dom}\left(\mathbf{W}_{f}\right)$, see Propositions 4.3 and 4.5 .

Homogeneous equations with initial values in $S\left(\mathbb{Q}_{p}^{n}\right)$

Let us define the function

$$
u(x, t)=Z_{t}(x) * u_{0}(x), \text { for } t \geqslant 0
$$

where $u_{0}(x):=Z_{0} * u_{0}=\left.\left(Z_{t}(x) * u_{0}(x)\right)\right|_{t=0}$.

Since $u_{0} \in S\left(\mathbb{Q}_{p}^{n}\right) \subset L^{\infty}\left(\mathbb{Q}_{p}^{n}\right)$ and $Z_{t}(x) \in L^{1}$ for $t>0$, then the convolution exists and it is a continuous function.

Lemma 4.2. Let $u(x, t)$ be defined for $t>0$ as in (8) and $u_{0} \in S\left(\mathbb{Q}_{p}^{n}\right)$ with the support of $\widehat{u}_{0}$ contained in $B_{N}$. Then the following assertions hold:

(i) $u(x, t)$ is continuously differentiable in time for $t \geqslant 0$, and its derivative is given by 


$$
\frac{\partial u(x, t)}{\partial t}=-k \mathscr{F}_{\xi \rightarrow x}^{-1}\left(e^{-k t A_{f}(\xi)} A_{f}(\xi) 1_{B_{N}}(\xi)\right) * u_{0}(x)
$$

(ii) $u(x, t) \in \operatorname{Dom}\left(\mathbf{W}_{f}\right)$ for any $t \geqslant 0$, and

$$
\left(\mathbf{W}_{f} u\right)(x, t)=k \mathscr{F}_{\xi \rightarrow x}^{-1}\left(e^{-k t A_{f}(\xi)} A_{f}(\xi) 1_{B_{N}}(\xi)\right) * u_{0}(x)
$$

Proof. The proof is similar in spirit to one given for Lemma 6.1 in [18].

The following result is a direct consequence of Lemma 4.2.

Proposition 4.3. If $u_{0} \in S\left(\mathbb{Q}_{p}^{n}\right)$ and $t \geqslant 0$, then $u(x, t)=Z_{t}(x) * u_{0}(x)$ is a solution of Cauchy problem (7).

Homogeneous equations with initial values in $L^{2}\left(\mathbb{Q}_{p}^{n}\right)$

Lemma 4.4. For any fixed $t \geqslant 0$, the operator $T(t): L^{2}\left(\mathbb{Q}_{p}^{n}\right) \rightarrow L^{2}\left(\mathbb{Q}_{p}^{n}\right)$ defined by the formula:

$$
T(t) u= \begin{cases}Z_{t} * u & \text { if } t>0 \\ u & \text { if } t=0\end{cases}
$$

is bounded.

Proof. For $t>0$, the result follows from Young's inequality by using the fact that $Z_{t} \in L^{1}\left(\mathbb{Q}_{p}^{n}\right)$, c.f. Theorem 3.9 (iii).

Proposition 4.5. The semigroup $(\mathscr{T}(t))_{t \geqslant 0}$ (see Proposition 4.1 (iii)) coincides with $(T(t))_{t \geqslant 0}$ (see (9)). Moreover, the Cauchy problem (7) is well-posed with solution $u(x, t)=Z_{t} * u_{0}$, for $t \geqslant 0$.

Proof. The operators $\mathscr{T}(t)$ and $T(t)$ are bounded and defined on the whole $L^{2}\left(\mathbb{Q}_{p}^{n}\right)\left(\right.$ Lemma 4.4) and by continuity we have that $\mathscr{T}(t)=T(t)$ on $L^{2}\left(\mathbb{Q}_{p}^{n}\right)$, since $\left.\mathscr{T}(t)\right|_{S}=\left.T(t)\right|_{S}$ (Proposition 4.3). The affirmation follows from results of the semigroup theory, see e.g., [28, Theorem 3.1.1], [27, Chap.2, Proposition 6.2] 


\section{Non-homogeneous equations}

Consider the following Cauchy problem:

$$
\begin{cases}\frac{\partial u}{\partial t}(x, t)+\mathbf{W}_{f} u(x, t)=g(x, t), & x \in \mathbb{Q}_{p}^{n}, t \in[0, T], T>0, \\ u(x, 0)=u_{0}(x), & u_{0}(x) \in \operatorname{Dom}\left(\mathbf{W}_{f}\right) .\end{cases}
$$

A solution of $(10)$ is given by a function $u(x, t) \in C\left([0, T), \operatorname{Dom}\left(\mathbf{W}_{f}\right)\right) \cap$ $C^{1}\left([0, T], L^{2}\left(\mathbb{Q}_{p}^{n}\right)\right)$ such that $u(x, t)$ satisfies equation $(10)$ for $t \in[0, T]$.

Theorem 4.6. Assume that $g \in C\left([0, \infty), L^{2}\left(\mathbb{Q}_{p}^{n}\right)\right) \cap L^{1}\left((0, \infty), \operatorname{Dom}\left(\mathbf{W}_{f}\right)\right)$ and let $u_{0} \in \operatorname{Dom}\left(\mathbf{W}_{f}\right)$ be given. The function

$$
u(x, t)=\int_{\mathbb{Q}_{p}^{n}} Z(x-\xi, t) u_{0}(\xi) \mathrm{d}^{n} \xi+\int_{0}^{t} \int_{\mathbb{Q}_{p}^{n}} Z(x-\xi, t-\theta) g(\xi, \theta) \mathrm{d}^{n} \xi \mathrm{d} \theta
$$

is the unique solution of (10).

Proof. The result follows by using some well-known results of the semigroup theory, see e.g., [28, Proposition 4.1.6] and Proposition 4.5.

Finally, we conclude that the heat kernel $Z_{t}(x)$ is a positive function.

Lemma 4.7. The $Z_{t}(x)$ function satisfies

$$
Z(x, t) \geqslant 0, \quad \text { for }(x, t) \in \mathbb{Q}_{p}^{n} \times(0, T] .
$$

Proof. It is sufficient to show that

$$
u(x, t)=\int_{\mathbb{Q}_{p}^{n}} Z(x-y, t) \varphi(y) \mathrm{d}^{n} y \geqslant 0,
$$

where $u(x, t)$ is the solution of Cauchy problem (7), with initial condition $u(x, 0)=\varphi(x) \geqslant 0$ and $\varphi \in S\left(\mathbb{Q}_{p}^{n}\right)$. Since

$$
u(x, t)=\int_{\mathbb{Q}_{p}^{n}} \chi_{p}(-x \cdot \xi) e^{-k t A_{f}(\xi)} \widehat{\varphi}(\xi) \mathrm{d}^{n} \xi
$$


by Riemann-Lebesgue lemma, we get

$$
u(x, t) \rightarrow 0 \text { as }\|x\|_{p} \rightarrow \infty .
$$

If $u(x, t)<0$, then there exist $x_{0} \in \mathbb{Q}_{p}^{n}$ and $t_{0} \in(0, T]$ such that

$$
\inf _{\substack{0 \leqslant t \leqslant T, x \in \mathbb{Q}_{p}^{n}}} u(x, t)=u\left(x_{0}, t_{0}\right)<0 .
$$

This implies that $\left(\mathbf{W}_{f} u\right)\left(x_{0}, t_{0}\right) \leqslant 0$, and $\frac{\partial u}{\partial t}\left(x_{0}, t_{0}\right) \leqslant 0$. Since $u(x, t)$ is the solution of Cauchy problem, we have that

$$
\frac{\partial u}{\partial t}(x, t)+\left(\mathbf{W}_{f} u\right)(x, t)=0 .
$$

Thus $\left(\mathbf{W}_{f} u\right)\left(x_{0}, t_{0}\right)=0$, then by (3), $u\left(x, t_{0}\right)$ is constant, and by (11) we have $u\left(x, t_{0}\right) \equiv 0$, which contradicts $(12)$.

\section{Markov Processes Over $\mathbb{Q}_{p}^{n}$}

We will denote by $\mathscr{B}\left(\mathbb{Q}_{p}^{n}\right)$ the $\sigma$-algebra of the Borel sets of $\mathbb{Q}_{p}^{n}$. Following the notation in [29], we denote by $\mathscr{B}_{0}$ the family of subsets of $\mathbb{Q}_{p}^{n}$ formed by finite unions of disjoint balls and the empty set. By Carathéodory's Theorem we conclude that if $\mu$ is a $\sigma$-finite measure on $\mathscr{B}_{0}$, then there is a unique measure on $\mathscr{B}\left(\mathbb{Q}_{p}^{n}\right)$ (also denoted by $\mu$ ) extending $\mu$.

Definition 5.1. For $E \in \mathscr{B}\left(\mathbb{Q}_{p}^{n}\right)$, we define

$$
p_{t}(x, E)= \begin{cases}T_{t} 1_{E}=Z_{t}(x) * 1_{E}(x) & \text { if } t>0 \\ 1_{E}(x) & \text { if } t=0 .\end{cases}
$$

Remark 3. By Lemma 4.7, we have that $p_{t}(x, \cdot) \geqslant 0$ for all $t \geqslant 0$ and $x \in \mathbb{Q}_{p}^{n}$, we also know that

$$
p_{t}(x, E)=\int_{\mathbb{Q}_{p}^{n}} \chi_{p}(-x \cdot \xi) e^{-k t A_{f}(\xi)} \widehat{1_{E}}(\xi) \mathrm{d}^{n} \xi, \quad \text { for } t>0 \text { and } x \in \mathbb{Q}_{p}^{n} .
$$

The following lemma is a consequence of above remark and Lemma 5 in [29].

Lemma 5.2. $p_{t}(x, \cdot), t \geqslant 0, x \in \mathbb{Q}_{p}^{n}$, is a measure on $\mathscr{B}\left(\mathbb{Q}_{p}^{n}\right)$.

Lemma 5.3. $p_{t}\left(x, \mathbb{Q}_{p}^{n}\right)=1$, for all $t \geqslant 0$ and $x \in \mathbb{Q}_{p}^{n}$. 
Proof. The result is a consequence of Definition 5.1 and Theorem 3.9 (ii).

Lemma 5.4. The function $p_{t}(\cdot, E)$ is a Borel measurable function for all $t>0$ and $E \in \mathscr{B}\left(\mathbb{Q}_{p}^{n}\right)$.

Proof. The proof is similar to Claim 2 in [29].

Lemma 5.5. The value $p_{0}(x,\{x\})=1$, for all $x \in \mathbb{Q}_{p}^{n}$.

Proof. This is a direct consequence of Definition 5.1.

Lemma 5.6 (The Chapman-Kolmogorov equation). For all $t, s \geqslant 0, x \in \mathbb{Q}_{p}^{n}$ and $E \in \mathscr{B}\left(\mathbb{Q}_{p}^{n}\right)$,

$$
p_{t+s}(x, E)=\int_{\mathbb{Q}_{p}^{n}} p_{t}\left(x, \mathrm{~d}^{n} y\right) p_{s}(y, E) .
$$

Proof. The proof is similar to Claim 4 in [29].

Lemma 5.7. The transition function $p_{t}(x, E)$ satisfies the following two conditions:

(i) $L(E)$. For each $s>0$ and compact $E \subset \mathbb{Q}_{p}^{n}$,

$$
\lim _{\|x\|_{p} \rightarrow \infty} \sup _{0 \leqslant t \leqslant s} p_{t}(x, E)=0
$$

(ii) $M(E)$. For each $\epsilon>0$ and compact $E \subset \mathbb{Q}_{p}^{n}$,

$$
\lim _{t \rightarrow 0^{+}} \sup _{x \in E} p_{t}\left(x, \mathbb{Q}_{p}^{n} \backslash B_{\epsilon}^{n}(x)\right)=0
$$

Proof. $\mathrm{L}(\mathrm{E})$. By using the compactness of $E$, there exists a ball $B_{N}^{n}$ such that $E \subset B_{N}^{n}$ and thus

$$
\begin{aligned}
0 \leqslant p_{t}(x, E) & \leqslant \int_{\mathbb{Q}_{p}^{n}} \chi_{p}(-x \cdot \xi) e^{-k t A_{f}(\xi) \widehat{1_{B_{N}^{n}}}(\xi) \mathrm{d}^{n} \xi} \\
& =p^{n N} \int_{\mathbb{Q}_{p}^{n}} \chi_{p}(-x \cdot \xi) e^{-k t A_{f}(\xi)} 1_{B_{-N}^{n}}(\xi) \mathrm{d}^{n} \xi \\
& =p^{n N} \mathscr{F}\left(e^{-k t A_{f}(\xi)} 1_{B_{-N}^{n}}(\xi)\right)(x),
\end{aligned}
$$


therefore

$$
0 \leqslant \lim _{\|x\|_{p} \rightarrow \infty} p_{t}(x, E) \leqslant \lim _{\|x\|_{p} \rightarrow \infty} p^{n N} \mathscr{F}\left(e^{-k t A_{f}(\xi)} 1_{B_{-N}^{n}}(\xi)\right)(x)=0
$$

where the last equality follows from Riemann-Lebesgue’s Lemma.

$\mathbf{M}(\mathrm{E})$. Note that $y \in \mathbb{Q}_{p}^{n} \backslash B_{\epsilon}^{n}(x) \Longleftrightarrow\|x-y\|_{p}>p^{\epsilon}$. Then by Fubini's Theorem we have

$$
\begin{aligned}
p_{t}\left(x, \mathbb{Q}_{p}^{n} \backslash B_{\epsilon}^{n}(x)\right) & =\int_{\|x-y\|_{p}>p^{\epsilon}} Z_{t}(x-y) \mathrm{d}^{n} y \\
& =1-\int_{\|x-y\|_{p} \leqslant p^{\epsilon}} Z_{t}(x-y) \mathrm{d}^{n} y \\
& =1-\int_{\|x-y\|_{p} \leqslant p^{\epsilon}}\left(\int_{\mathbb{Q}_{p}^{n}} \chi_{p}(-(x-y) \cdot \xi) e^{-k t A_{f}(\xi)} \mathrm{d}^{n} \xi\right) \mathrm{d}^{n} y \\
& =1-\int_{\mathbb{Q}_{p}^{n}} e^{-k t A_{f}(\xi)}\left(\int_{\|z\|_{p} \leqslant p^{\epsilon}} \chi_{p}(z \cdot \xi) \mathrm{d}^{n} z\right) \mathrm{d}^{n} \xi \\
& =1-p^{n \epsilon} \int_{\|\xi\|_{p} \leqslant p^{-\epsilon}} e^{-k t A_{f}(\xi)} \mathrm{d}^{n} \xi,
\end{aligned}
$$

now by Lebesgue's Dominated Convergence Theorem,

$$
\begin{aligned}
\lim _{t \rightarrow 0^{+}} \sup _{x \in E} p_{t}\left(x, \mathbb{Q}_{p}^{n} \backslash B_{\epsilon}^{n}(x)\right) & =\lim _{t \rightarrow 0^{+}} \sup _{x \in E}\left(1-p^{n \epsilon} \int_{\|\xi\| \leqslant p^{-\epsilon}} e^{-k t A_{f}(\xi)} \mathrm{d}^{n} \xi\right) \\
& =1-p^{n \epsilon} \int_{\|\xi\| \leqslant p^{-\epsilon}} \mathrm{d}^{n} \xi \\
& =0 .
\end{aligned}
$$

Theorem 5.8. The function $p_{t}(x, \cdot)$ is the transition density of a time and space homogeneous Markov process $\mathfrak{T}(t, \omega)$, which is bounded, right-continuous, and has no discontinuities other than jumps.

Proof. It is sufficient to use Lemma 5.7 and [30, Theorem 3.6] together with the observation that $\left(\mathbb{Q}_{p}^{n},\|x\|_{p}\right)$ is a locally compact Hausdorff space with a countable base. 


\section{The First Passage Time Problem}

In this section we study the first passage time problem of a path of the Markov process $\mathfrak{T}(t, \omega)$.

By Proposition 4.3 the function

$$
u(x, t)=Z_{t}(x) * \Omega\left(\|x\|_{p}\right)=\int_{\mathbb{Z}_{p}^{n}} \chi_{p}(x \cdot \xi) e^{-k t A_{f}(\xi)} \mathrm{d}^{n} \xi,
$$

is a classical solution of

$$
\left\{\begin{array}{l}
\frac{\partial u}{\partial t}(x, t)=-\left(\mathbb{W}_{f} u\right)(x, t), \quad x \in \mathbb{Q}_{p}^{n}, t \geqslant 0, \\
u(x, 0)=\Omega\left(\|x\|_{p}\right) .
\end{array}\right.
$$

Now, for $E \in \mathscr{B}\left(\mathbb{Q}_{p}^{n}\right)$ we define

$$
q_{t}(x, E)= \begin{cases}\left(u(\cdot, t) * 1_{E}\right)(x), & \text { for } t>0 \\ 1_{E}(x), & t=0 .\end{cases}
$$

This $q_{t}(x, E)$ is a transition function of a Markov process $\mathfrak{T}(t, \omega)$. Thus, there exists a probability space $(\mathfrak{Y}, \mathfrak{F}, P)$ and $\mathfrak{T}(t, \cdot):(\mathfrak{Y}, \mathfrak{F}, P) \rightarrow\left(\mathbb{Q}_{p}^{n}, \mathscr{B}\left(\mathbb{Q}_{p}^{n}, \mathrm{~d}^{n} x\right)\right)$ is a random variable for each $t>0$. Note that

$$
P\left(\left\{\omega \in \mathfrak{Y}: \mathfrak{T}(0, \omega) \in \mathbb{Z}_{p}^{n}\right\}\right)=q_{0}\left(0, \mathbb{Z}_{p}^{n}\right)=1 .
$$

Definition 6.1. The random variable $\tau_{\mathbb{Z}_{p}^{n}}(\omega): \mathfrak{Y} \rightarrow \mathbb{R}_{+} \cup\{+\infty\}$ defined by $\inf \left\{t>0 \mid \mathfrak{T}(t, \omega) \in \mathbb{Z}_{p}^{n}\right.$ and there exists $t^{\prime}$ s.t. $0<t^{\prime}<t$ and $\left.\mathfrak{T}\left(t^{\prime}, \omega\right) \notin \mathbb{Z}_{p}^{n}\right\}$ is called the first passage time of a path of the random process $\mathfrak{T}(t, \omega)$ entering the domain $\mathbb{Z}_{p}^{n}$.

Lemma 6.2. If $u(x, t)=Z_{t}(x) * \Omega\left(\|x\|_{p}\right)$ for $t \geqslant 0$, then $u(x, t)$ is pointwise differentiable in $t$, furthermore its derivative is given by

$$
\frac{\partial u}{\partial t}(x, t)=-k \int_{\mathbb{Z}_{p}^{n}} \chi_{p}(-x \cdot \xi) e^{-k t A_{f}(\xi)} A_{f}(\xi) \mathrm{d}^{n} \xi, \text { for } t \geqslant 0 .
$$

Proof. The proof is a straightforward application of Lebesgue's Dominated Convergence Theorem. 
Lemma 6.3. If $C:=\left(k \int_{\mathbb{Q}_{p}^{n} \backslash \mathbb{Z}_{p}^{n}} \frac{1}{|f(y)|_{p}^{\alpha+\frac{n}{d}}} \mathrm{~d}^{n} y\right) \leqslant 1$, then the probability density function for a path of $\mathfrak{T}(t, \omega)$ to enter into $\mathbb{Z}_{p}^{n}$ at the time $t$, with initial condition $\mathfrak{T}(0, \omega) \in \mathbb{Z}_{p}^{n}$ is given by

$$
g(t)=k \int_{\mathbb{Q}_{p}^{n} \backslash \mathbb{Z}_{p}^{n}} \frac{u(y, t)}{|f(y)|^{\alpha+\frac{n}{d}}} \mathrm{~d}^{n} y .
$$

Proof. The survival probability $S(t)$ is the probability that a path of $\mathfrak{T}(t, \omega)$ remains in $\mathbb{Z}_{p}^{n}$ at the time $t$, i.e.

$$
S(t):=S_{\mathbb{Z}_{p}^{n}}(t)=\int_{\mathbb{Z}_{p}^{n}} u(x, t) \mathrm{d}^{n} x
$$

By using Lemma 6.2 and Lebesgue's Dominated Convergence Theorem,

$$
\begin{aligned}
S^{\prime}(t) & =\int_{\mathbb{Z}_{p}^{n}} \frac{\partial u(x, t)}{\partial t} \mathrm{~d}^{n} x=k \int_{\mathbb{Z}_{p}^{n}} \int_{\mathbb{Q}_{p}^{n}} \frac{u(x-y, t)-u(x, t)}{|f(y)|_{p}^{\alpha+\frac{n}{d}}} \mathrm{~d}^{n} y \mathrm{~d}^{n} x \\
& =k \int_{\mathbb{Z}_{p}^{n} \mathbb{Z}_{p}^{n}} \frac{u(x-y, t)-u(x, t)}{|f(y)|_{p}^{\alpha+\frac{n}{d}}} \mathrm{~d}^{n} y \mathrm{~d}^{n} x \\
& +k \int_{\mathbb{Z}_{p}^{n}} \int_{\mathbb{Q}_{p}^{n} \backslash \mathbb{Z}_{p}^{n}} \frac{u(x-y, t)-u(x, t)}{|f(y)|_{p}^{\alpha+\frac{n}{d}}} \mathrm{~d}^{n} y \mathrm{~d}^{n} x .
\end{aligned}
$$

Now from equation (13) we have that $u(x-y, t)-u(x, t) \equiv 0$ for $x, y \in \mathbb{Z}_{p}^{n}$, in effect

$$
\begin{aligned}
u(x-y, t) & =\int_{\mathbb{Z}_{p}^{n}} \chi_{p}(-(x-y) \cdot \xi) e^{-k t A_{f}(\xi)} \mathrm{d}^{n} \xi \\
& =\int_{\mathbb{Z}_{p}^{n}} e^{-k t A_{f}(\xi)} \mathrm{d}^{n} \xi \\
& =u(x, t),
\end{aligned}
$$


consequently,

$$
\begin{aligned}
S^{\prime}(t) & =k \int_{\mathbb{Z}_{p}^{n}} \int_{\mathbb{Q}_{p}^{n} \backslash \mathbb{Z}_{p}^{n}} \frac{u(x-y, t)-u(x, t)}{|f(y)|_{p}^{\alpha+\frac{n}{d}}} \mathrm{~d}^{n} y \mathrm{~d}^{n} x . \\
& =-k \int_{\mathbb{Z}_{p}^{n}} \int_{\mathbb{Q}_{p}^{n} \backslash \mathbb{Z}_{p}^{n}} \frac{u(x, t)}{|f(y)|_{p}^{\alpha+\frac{n}{d}}} \mathrm{~d}^{n} y \mathrm{~d}^{n} x+k \int_{\mathbb{Z}_{p}^{n}} \int_{\mathbb{Q}_{p}^{n} \backslash \mathbb{Z}_{p}^{n}} \frac{u(x-y, t)}{|f(y)|_{p}^{\alpha+\frac{n}{d}}} \mathrm{~d}^{n} y \mathrm{~d}^{n} x \\
& =-k \int_{\mathbb{Q}_{p}^{n} \backslash \mathbb{Z}_{p}^{n}} \frac{1}{|f(y)|_{p}^{\alpha+\frac{n}{d}}} \mathrm{~d}^{n} y \int_{\mathbb{Z}_{p}^{n}} u(x, t) \mathrm{d}^{n} x+k \int_{\mathbb{Z}_{p}^{n}} \int_{\mathbb{Q}_{p}^{n} \backslash \mathbb{Z}_{p}^{n}} \frac{u(x-y, t)}{|f(y)|_{p}^{\alpha+\frac{n}{d}}} \mathrm{~d}^{n} y \mathrm{~d}^{n} x .
\end{aligned}
$$

Now if $x \in \mathbb{Z}_{p}^{n}$ and $y \in \mathbb{Q}_{p}^{n} \backslash \mathbb{Z}_{p}^{n}$, then

$$
u(x-y, t)=\int_{\mathbb{Z}_{p}^{n}} \chi_{p}(-x \cdot \xi) \chi_{p}(y \cdot \xi) e^{-k t A_{f}(\xi)} \mathrm{d}^{n} \xi=u(y, t),
$$

therefore

$$
S^{\prime}(t)=\left(-k \int_{\mathbb{Q}_{n}^{p} \backslash \mathbb{Z}_{p}^{n}} \frac{1}{|f(y)|_{p}^{\alpha+\frac{n}{d}}} \mathrm{~d}^{n} y\right) S(t)+\left(k \int_{\mathbb{Q}_{p}^{n} \backslash \mathbb{Z}_{p}^{n}} \frac{u(y, t)}{|f(y)|_{p}^{\alpha+\frac{n}{d}}} \mathrm{~d}^{n} y\right) .
$$

On the other hand, since there are no external or internal sources,

$$
\begin{aligned}
S^{\prime}(t) & =\left\{\begin{array}{c}
\text { Probability that a path of } \\
\mathfrak{T}(t, \omega) \text { goes back to } \mathbb{Z}_{p}^{n} \text { at time } t
\end{array}\right\}-\left\{\begin{array}{l}
\text { Probability that a path of } \\
\mathfrak{T}(t, \omega) \text { exits } \mathbb{Z}_{p}^{n} \text { at time } t
\end{array}\right\} \\
& =g(t)-C \cdot S(t), \quad \text { with } 0<C \leqslant 1 .
\end{aligned}
$$

Finally, comparing (15) and the last equality we get the desired formula.

Proposition 6.4. The probability density function $f(t)$ of the random variable $\tau(\omega)$ satisfies the non-homogeneous Volterra equation of second kind

$$
g(t)=\int_{0}^{\infty} g(t-\tau) f(\tau) \mathrm{d} \tau+f(t) .
$$

Proof. The result follows from Lemma 6.3 by using the argument given in the proof of Theorem 1 in [2]. 
Lemma 6.5. For all $s, k>0$, and $z \in \mathbb{Z}_{p}^{n}$, with $\|z\|_{p}=1$, we have

$$
\frac{1-p^{-n}}{s+C_{2} k} \leqslant \int_{\|z\|_{p}=1} \frac{1}{s+k A_{f}(z)} \mathrm{d}^{n} z \leqslant \frac{1-p^{-n}}{s+C_{1} k}
$$

Proof. It is consequence of Lemma 3.7.

Proposition 6.6. The Laplace transform $G(s)$ of $g(t)$ is given by

$$
G(s)=k \int_{\mathbb{Q}_{p}^{n} \backslash \mathbb{Z}_{p}^{n}} \frac{1}{|f(y)|_{p}^{\alpha+\frac{n}{d}}} \int_{\mathbb{Z}_{p}^{n}} \frac{\chi_{p}(-\xi \cdot y)}{s+k A_{f}(\xi)} \mathrm{d}^{n} \xi \mathrm{d}^{n} y .
$$

Proof. For $s \in \mathbb{C}$ with $\operatorname{Re}(s)>0$, we have $e^{-s t}|f(y)|_{p}^{-\alpha-n / d} e^{-t k A_{f}(\xi)} \Omega\left(\|\xi\|_{p}\right) \in$ $L^{1}\left((0, \infty) \times\left(\mathbb{Q}_{p}^{n} \backslash \mathbb{Z}_{p}^{n}\right) \times \mathbb{Q}_{p}^{n}, \mathrm{~d} t \mathrm{~d}^{n} y \mathrm{~d}^{n} \xi\right)$ and the formula follows now from Lemma 6.3 and equation (13) by using Fubini's Theorem.

Definition 6.7. We say that $\mathfrak{T}(t, \omega)$ is recurrent with respect to $\mathbb{Z}_{p}^{n}$ if

$$
P\left(\left\{\omega \in \mathfrak{Y}: \tau_{\mathbb{Z}_{p}^{n}}(\omega)<\infty\right\}\right)=1
$$

Otherwise, we will say that $\mathfrak{T}(t, \omega)$ is transient with respect to $\mathbb{Z}_{p}^{n}$.

Proposition 6.8. The Laplace transform $G(s)$ of $g(t)$ is given by the expression $G(s)=G_{1}(s)+G_{2}(s)$, where

$$
G_{1}(s)=k \sum_{\gamma=1}^{\infty} \sum_{\delta=0}^{\gamma-1} p^{-d \alpha \gamma-n \delta} \int_{\|x\|_{p}=1} \frac{1}{|f(x)|_{p}^{\alpha+\frac{n}{d}}} \int_{\|z\|_{p}=1} \frac{\chi_{p}\left(-p^{\delta-\gamma} z \cdot x\right)}{s+k p^{-d \alpha \delta} A_{f}(z)} \mathrm{d}^{n} z \mathrm{~d}^{n} x
$$

and

$$
G_{2}(s)=k \sum_{\gamma=1}^{\infty} \sum_{\delta=\gamma}^{\infty} p^{-d \alpha \gamma-n \delta} \int_{\|x\|_{p}=1} \frac{1}{|f(x)|_{p}^{\alpha+\frac{n}{d}}} \int_{\|z\|_{p}=1} \frac{1}{s+k p^{-d \alpha \delta} A_{f}(z)} \mathrm{d}^{n} z \mathrm{~d}^{n} x
$$

Proof. A direct calculation shows that 


$$
\begin{aligned}
G(s) & =k \int_{\mathbb{Q}_{p}^{n} \backslash \mathbb{Z}_{p}^{n}} \frac{1}{|f(y)|_{p}^{\alpha+\frac{n}{d}}} \int_{\mathbb{Z}_{p}^{n}} \frac{\chi_{p}(-\xi \cdot y)}{s+k A_{f}(\xi)} \mathrm{d}^{n} \xi \mathrm{d}^{n} y \\
& =k \sum_{\gamma=1}^{\infty} \sum_{\delta=0}^{\infty} \int_{\|y\|_{p}=p^{\gamma}} \frac{1}{|f(y)|_{p}^{\alpha+\frac{n}{d}}} \int_{\|\xi\|_{p}=p^{-\delta}} \frac{\chi_{p}(-\xi \cdot y)}{s+k A_{f}(\xi)} \mathrm{d}^{n} \xi \mathrm{d}^{n} y \\
& =k \sum_{\gamma=1}^{\infty} \sum_{\delta=0}^{\infty} \int_{\left\|p^{\gamma} y\right\|_{p}=1} \frac{1}{|f(y)|_{p}^{\alpha+\frac{n}{d}}} \int_{\left\|p^{-\delta \xi}\right\|_{=1}} \frac{\chi_{p}(-\xi \cdot y)}{s+k A_{f}(\xi)} \mathrm{d}^{n} \xi \mathrm{d}^{n} y \\
& =k \sum_{\gamma=1}^{\infty} \sum_{\delta=0}^{\infty} p^{-d \alpha \gamma-n \delta} \int_{\|x\|_{p}=1} \frac{1}{|f(x)|_{p}^{\alpha+\frac{n}{d}}} \int_{\|z\|_{p}=1} \frac{\chi_{p}\left(-p^{\delta-\gamma} z \cdot x\right)}{s+k p^{-d \alpha \delta} A_{f}(z)} \mathrm{d}^{n} z \mathrm{~d}^{n} x .
\end{aligned}
$$

From where we obtain

$$
\begin{aligned}
G(s)= & k \sum_{\gamma=1}^{\infty} \sum_{\delta=0}^{\gamma-1} p^{-d \alpha \gamma-n \delta} \int_{\|x\|_{p}=1} \frac{1}{|f(x)|_{p}^{\alpha+\frac{n}{d}}} \int_{\|z\|_{p}=1} \frac{\chi_{p}\left(-p^{\delta-\gamma} z \cdot x\right)}{s+k p^{-d \alpha \delta} A_{f}(z)} \mathrm{d}^{n} z \mathrm{~d}^{n} x \\
& +k \sum_{\gamma=1}^{\infty} \sum_{\delta=\gamma}^{\infty} p^{-d \alpha \gamma-n \delta} \int_{\|x\|_{p}=1} \frac{1}{|f(x)|_{p}^{\alpha+\frac{n}{d}}} \int_{\|z\|_{p}=1} \frac{1}{s+k p^{-d \alpha \delta} A_{f}(z)} \mathrm{d}^{n} z \mathrm{~d}^{n} x \\
= & : G_{1}(s)+G_{2}(s) .
\end{aligned}
$$

Theorem 6.9. $\mathfrak{T}(t, \omega)$ is recurrent with respect to $\mathbb{Z}_{p}^{n}$ for $\alpha \geqslant \frac{n}{d}$, and transient with respect to $\mathbb{Z}_{p}^{n}$ for $\alpha<\frac{n}{d}$.

Proof. By taking Laplace transform in (16), we get

$$
F(s):=\mathscr{L}\{f(t)\}(s)=\frac{G(s)}{1+G(s)},
$$

where $G(s):=\mathscr{L}\{g(t)\}(s)$. Hence

$$
F(0)=\int_{0}^{\infty} f(t) \mathrm{d} t=1-\frac{1}{1+G(0)}
$$

In order to get the recurrence for $\mathfrak{T}(t, \omega)$ is sufficient to show that

$$
G(0)=\lim _{s \rightarrow 0} G(s)=\infty
$$


and to prove that it is transient that

$$
G(0)=\lim _{s \rightarrow 0} G(s)<\infty
$$

If we take $s=p^{-d \alpha \delta}=p^{-d \alpha \gamma}$, then $s \rightarrow 0^{+} \Leftrightarrow \gamma \rightarrow \infty(\delta=\gamma)$. Now, taking only the first term of $G_{2}(s)$ we conclude that

$$
\begin{aligned}
G_{2}(s) & >\int_{\|x\|_{p}=1} \frac{\mathrm{d}^{n} x}{|f(x)|_{p}^{\alpha+\frac{n}{d}}} \sum_{\delta=1}^{\infty} \frac{p^{-d \alpha-n \delta}}{p^{-d \alpha \delta}} \int_{\|z\|_{p}=1} \frac{1}{1+k A_{f}(z)} \mathrm{d}^{n} z \\
& =p^{-d \alpha} \int_{\|x\|_{p}=1} \int_{\|z\|_{p}=1} \frac{\mathrm{d}^{n} z \mathrm{~d}^{n} x}{|f(x)|_{p}^{\alpha+\frac{n}{d}}\left(1+k A_{f}(z)\right)} \sum_{\delta=1}^{\infty} p^{(-n+\alpha d) \delta}
\end{aligned}
$$

and $G_{2}(s)$ diverges if $\alpha \geqslant \frac{n}{d}$. Since $G_{1}\left(p^{-d \alpha \delta}\right)<\infty$, if $\alpha \geqslant \frac{n}{d}$, then the value $G(s)=G_{1}(s)+G_{2}(s)$ diverges.

Finally, using Lemma 6.5, we have that

$$
\begin{aligned}
|G(s)| & \leqslant k \sum_{\gamma=1}^{\infty} \sum_{\delta=0}^{\infty} p^{-d \alpha \gamma-n \delta} \int_{\|x\|_{p}=1} \frac{1}{|f(x)|_{p}^{\alpha+\frac{n}{d}}} \int_{\|z\|_{p}=1} \frac{1}{s+k p^{-d \alpha \delta} A_{f}(z)} \mathrm{d}^{n} z \mathrm{~d}^{n} x \\
& \leqslant k \frac{1-p^{-n}}{C_{1} k} \int_{\|x\|_{p}=1} \frac{\mathrm{d}^{n} x}{|f(x)|_{p}^{\alpha+\frac{n}{d}}} \sum_{\gamma=1}^{\infty} \sum_{\delta=0}^{\infty} p^{-d \alpha \gamma-n \delta+d \alpha \delta} .
\end{aligned}
$$

Therefore $\lim _{s \rightarrow 0^{+}} G(s)<\infty$ for $\alpha<\frac{n}{d}$.

\section{Acknowledgements}

The first a uthor was partially supported by the research p roject Blow-up phenomenon for a p-adic nonlocal semilinear diffusion equation, with ID-PPTA: 00008213 of the Faculty of Sciences of the Pontificia Universidad Javeriana, Bogotá, Colombia.

\section{Conflict of Interest}

The authors declare no conflict of interest. 


\section{References}

[1] Avetisov VA, Bikulov A Kh. Ultrametricity of fluctuation dynamic mobility of protein molecules, Proceedings of the Steklov Institute of Mathematics, 265: 75-81, 2009. (Russian), Trudy Matematicheskogo Instituta imeni V.A. Steklova, 265: 82-89, 2009.

doi: 10.1134/S0081543809020060

[2] Avetisov VA, Bikulov AKh, Zubarev AP. First passage time distribution and the number of returns for ultrametric random walks, Journal of Physics A: Mathematical and Theoretical, 42(8): 085003, 2009.

doi: 10.1088/1751-8113/42/8/085003

[3] Avetisov VA, Bikulov AKh, Osipov VA. p-adic models of ultrametric diffusion in conformational dynamics of macromolecules. (Russian), Trudy Matematicheskogo Instituta imeni V.A. Steklova, 245(2): 55-64, 2004

[4] Avetisov VA, Bikulov AKh, Osipov VA. p-adic description of characteristic relaxation in complex systems, Journal of Physics $A$ : Mathematical and General, 36 (15): 4239-4246, 2003.

doi: 10.1088/0305-4470/36/15/301

[5] Avetisov VA, Bikulov AH, Kozyrev SV, Osipov VA. p-adic models of ultrametric diffusion constrained by hierarchical energy landscapes, Journal of Physics A: Mathematical and General, 35(2): 177-189, 2002.

doi: $10.1088 / 0305-4470 / 35 / 2 / 301$

[6] Avetisov VA, Bikulov AKh, Kozyrev SV. Description of logarithmic relaxation by a model of a hierarchical random walk. (Russian), Doklady Akademii Nauk, 368(2): 164-167, 1999.

[7] Dragovich B, Khrennikov AYu, Kozyrev SV, Volovich IV. On $p$-adic mathematical physics, $p$-Adic Numbers Ultrametric Analysis and Applications, 1(1): 1-17, 2009.

doi: 10.1134/S2070046609010014

[8] Khrennikov AYu, Kozyrev SV. p-adic pseudodifferential operators and analytic continuation of replica matrices, Theoretical and Mathematical Physics, 144(2): 1166-1170, 2005.

doi: $10.4213 / \operatorname{tmf1858}$ 
[9] Mezard M, Parisi G, Virasoro MA. Spin glass theory and beyond, In World Scientific Lecture Notes in Physics, 1987.

doi: 10.1142/0271

[10] Varadarajan VS. Path integrals for a class of $p$-adic Schrodinger equations, Letters in Mathematical Physics, 39 (2): 97-106, 1997.

doi: 10.1023/A:1007364631796

[11] Vladimirov VS, Volovich IV, Zelenov EI. p-analysis and mathematical physics, World Scientific, 1994.

[12] Zúñiga-Galindo WA. Parabolic equations and Markov processes over $p$-adic fields, Potential Analysis, 28 (2): 185-200, 2008.

doi: 10.1007/s11118-007-9072-2

[13] Galeano-Peñaloza J, Zúñiga-GalindoWA. Pseudo-differential operators with semi-quasielliptic symbols over $p$-adic fields, Journal of Mathematical Analysis and Applications, 386 (1): 32-49, 2012.

doi: 10.1016/j.jmaa.2011.07.040

[14] KarwowskiW. Diffusion processes with ultrametric jumps, Reports on Mathematical Physics, 60 (2): 221-235, 2007.

doi: 10.1016/S0034-4877(07)00025-0

[15] Rammal R, Toulouse G, Virasoro MA. Ultrametricity for physicists, Reviews of Modern Physics, 58 (3): 765-788, 1986.

doi: 10.1103/RevModPhys.58.765

[16] Kochubei AN. Pseudo-differential equations and stochastics over non-Archimedean fields, Monographs and Textbooks in Pure and Applied Mathematics, 244, Marcel Dekker, Inc., New York, 2001.

[17] Chacón-Cortés LF, Zúñiga-Galindo WA. Non-local operators, non-Archimedean parabolic-type equations with variable coefficients and Markov processes, Publications of the Research Institute for Mathematical Sciences PRIMS, 51 (2): 289-317, 2015.

doi: 10.4171/PRIMS/156

[18] Chacón-Cortés LF, Zúñiga-GalindoWA. Nonlocal operators, parabolictype equations, and ultrametric random walks, Journal of Mathematical Physics, 54 (11): 113503, 2013.

doi: $10.1063 / 1.4828857$ 
[19] Casas-Sánchez OF, Galeano-Peñaloza J, Rodríguez-Vega JJ. Parabolictype pseudodifferential equations with elliptic symbols in dimension 3 over $p$-adics, $p$-Adic Numbers Ultrametric Analysis and Applications, 7(1): 1-16, 2015.

doi: 10.1134/S207004661501001X

[20] Chacón-Cortés LF. The problem of the first passage time for some elliptic pseudodiffe-rential operators over the $p$-adics, Revista Colombiana de Matemáticas, 48 (2): 191-209, 2014.

doi: 10.15446/recolma.v48n2.54124

[21] Casas-Sánchez OF, Galeano-Peñaloza J, Rodríguez-Vega JJ. The problem of the first return attached to a pseudodifferential operator in dimension 3, Revista Integración. Temas Matemáticos, 33(2): 107-119, 2015.

doi: 10.18273/revint.v33n2-2015002

[22] Casas-Sánchez OF, Zúñiga-Galindo WA. p-adic elliptic quadratic forms, parabolic-type pseudodifferential equations with variable coefficients and Markov processes, $p$-Adic Numbers Ultrametric Analysis and Applications, 6 (1): 1-20, 2014.

doi: 10.1134/S2070046614010014

[23] Albeverio S, Khrennikov AYu, Shelkovich VM. Theory of $p$-adic distributions: linear and nonlinear models. Cambridge University Press, 2010.

[24] Taibleson MH. Fourier analysis on local fields, Princeton University Press, 1975.

[25] Zúñiga-Galindo WA. Pseudodifferential Equations Over NonArchimedean Spaces, Lectures Notes in Mathematics vol. 2174, Springer, 2016.

[26] Berg C and Forst G. Potential theory on locally compact abelian groups, Springer-Verlag, New York, 1975.

[27] Engel K-J, Nagel R. One-Parameter Semigroups for Linear Evolution Equations. Springer-Verlag, 2000.

[28] Cazenave Thierry, Haraux Alain: An introduction to semilinear evolution equations. Oxford University Press, 1998. 
[29] Torresblanca-Badillo A, Zúñiga-Galindo WA. Ultrametric Diffusion, Exponential Landscapes, and the First Passage Time Problem, Acta Applicandae Mathematicae, 157(1): 93-116, 2018.

doi: 10.1007/s10440-018-0165-2

[30] Dynkin EB. Markov processes. Vol. I. Springer-Verlag, 1965

\section{Funciones no radiales, operadores no locales y procesos de Markov sobre números $p$-ádicos}

Resumen: El objetivo principal de este artículo es estudiar una nueva clase de operadores no locales y el problema de Cauchy para ciertas ecuaciones diferenciales de tipo parabólico asociadas naturalmente con ellos. Las soluciones fundamentales de estas ecuaciones son funciones de transición de procesos de Markov en un espacio vectorial $n$-dimensional sobre los números $p$-ádicos. También se estudian algunas propiedades de estos procesos de Markov, incluyendo el problema del tiempo del primer retorno.

Palabras claves: procesos de Markov; análisis no arquimediano; operadores no locales; números $p$-ádicos.

\section{Funçóes não radiais, operadores não-locais e processos de Markov sobre números $p$-ádicos}

Resumo: O principal objetivo deste artigo é estudar uma nova classe de operadores não-locais e o problema de Cauchy para certas equações pseudodiferenciais de tipo parabólico naturalmente associadas a elas. As soluções fundamentais dessas equações são funções de transição dos processos de Markov em um espaço vetorial $n$-dimensional sobre os números $p$-ádicos. Também estudamos algumas propriedades desses processos de Markov, incluindo o problema do tempo do primeiro retorno.

Palavras-chave: processos de Markov; análise não-arquimediana; operadores não-locais; números $p$-ádicos. 


\section{Oscar Francisco Casas Sánchez}

Is Associate Professor in the Universidad Pedagógica y Tecnológica de Colombia. Received his Ph.D degree in Mathematics in 2014 from Universidad Nacional de Colombia, he is Mathematician and M. Sc. in Mathematics of the same university. His research interest is Non-Archimedean analysis.

ORCID: 0000-0002-8725-5385

\section{Leonardo Fabio Chacón Cortés}

Ph.D. Assistant Professor, Department of Mathematics, Pontifical Xavierian University, Bogotá D.C., Colombia. Mainly interested in the areas of mathematical analysis, partial differential equations, $p$-adic analysis, stochastic processes among others.

ORCID: 0000-0003-1335-7941 